\title{
Précis of The Red and the Real: An Essay on Color Ontology
}

\author{
Jonathan Cohen* \\ "I can't explain myself, I'm afraid, sir," said Alice, "because I'm not myself, you see." \\ "I don't see," said the Caterpillar. \\ — Lewis Carroll, Alice's Adventures in Wonderland, 5.
}

The Red and the Real is aimed at understanding what kinds of properties the colors are. It offers two (compatible) answers. The first, whose elaboration and defense occupies Parts I and II, is that colors are relational properties - properties constituted in terms of relations to subjects and perceptual circumstances. The second, developed in Part III, is that the particular relational properties to which colors are identical are functional roles: viz., that colors are identical with the functional roles of disposing their bearers to look colored to subjects in circumstances.

In this précis I outline the main positions and arguments of the book.

\section{Part I: The Case for Color Relationalism}

To say that colors are relational properties is to say that things have their colors by being appropriately related to (actual or possible) perceivers (inter alia). Plausibly, things exemplify properties like being a sister or being a teacher in virtue of the relations they bear to other individuals. The color relationalist holds that, likewise, things exemplify colors not in virtue of their material makeups, interactions with light, primitive extra properties, etc., but in virtue of the relations they bear to perceivers. Part I of The Red and the Real is devoted to arguing for this view.

My case for relationalism revolves around a traditional, non-deductive, empirical argument based on perceptual variation. While the argument can

* Department of Philosophy, University of California, San Diego, 9500 Gilman Drive, La Jolla, CA 92093-0119, joncohen@aardvark.ucsd.edu 
be generalized widely (see below), it is best initially appreciated in a single case such as that involving the central patches in figure 1. Though these two

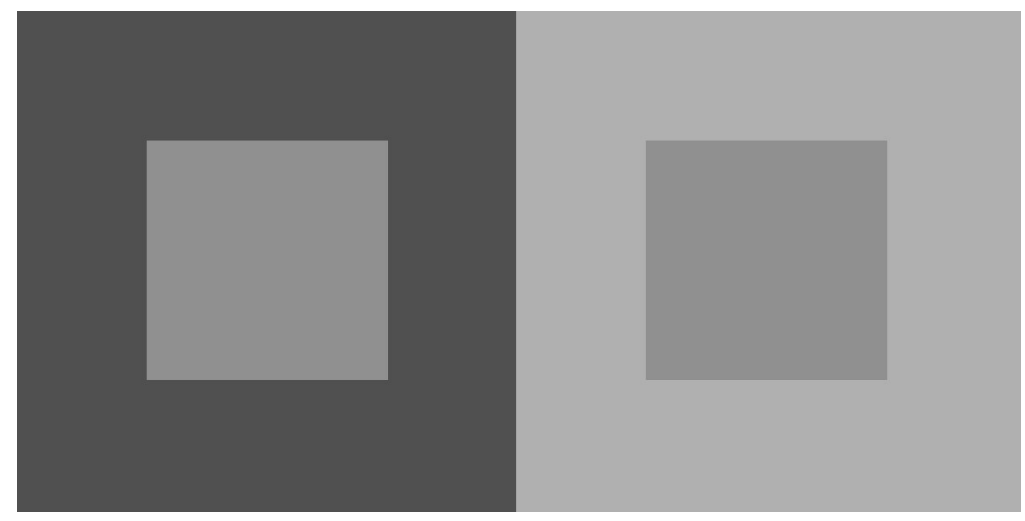

Figure 1: The two center gray squares have equal reflectances, but the one against the lighter background appears darker than the one against the darker background.

central patches are qualitatively identical in their non-relational properties, they look different (in color) depending on the surround against which they are placed. Assuming, standardly, that the way the patches look represents their colors, it follows that the visual system represents the colors of the patches differently as a function of the surround. If so, we can ask: which, if any of the (psychophysically distinguishable) representations of the patches is veridical? ? $^{1}$ The logically possible answers are: neither, the first to the exclusion of the second, the second to the exclusion of the first, or both. I suggest that the first option (neither right) is unacceptably skeptical/revisionary, and that the second and third answers (one representation exclusively) are unacceptably arbitrary - that it is hard to imagine a well-motivated, principled, and non-question begging reason to believe that either representation is uniquely veridical. Assuming we want to avoid both revisionary skepticism and ad hoc stipulation when possible, this suggests that, ceteris paribus, we should prefer the view that both representations are veridical. My further suggestion is that we should endorse color relationalism because it gives the best way of understanding how this answer could be true. For relationalism allows us to say that the patch has two compatible colors: it is (simultaneously, all over) light grey with respect to the first perceptual circumstance, and dark grey with respect to the second ${ }^{2}$ Color relationalism is attractive because it allows us to avoid a hard choice between unpalatable alternatives.

\footnotetext{
${ }^{1}$ The question here is metaphysical, not epistemic: it is not 'how do ordinary perceivers know which of the perceptual effects veridically represents the patch's color?', but 'what makes it the case that one of the perceptual effects (as opposed to others) veridically represents the patch's color?'.

${ }^{2}$ Analogy: a single individual can be a sister of you, and, simultaneously, not a sister of me.
} 
As indicated above, this argumentative pattern generalizes widely to interspecies, intrapersonal, and interpersonal instances of perceptual variation.

Interspecies: Perceivers of different species differ considerably in their color vision: the chromatic effects that a single stimulus has on these perceivers vary widely as a function of many parameters of their visual systems - retinal cone type populations (and population ratios), cone tuning curves, macular and lens pigmentation, and on and on. While there may be principled grounds for saying that some such variants represent the stimulus color erroneously, there remains significant variation between variants in organisms that pass standard comparative psychophysical criteria for normal color vision; as such, it would be objectionably ad hoc to treat these variants as systematically misrepresenting the colors of objects. The relationalist holds that all of these variants are veridical, thereby avoiding undue skepticism and ad hoc stipulation, by holding that the stimulus exemplifies (simultaneously, all over) one color with respect to perceivers of kind $K_{1}$, another color with respect to perceivers of kind $K_{2}$, and so on.

Interpersonal: Human perceivers differ considerably in their color vision: the chromatic effects of a single stimulus on these viewers vary widely as a function of many parameters of their visual systems - retinal cone populations (and population ratios), cone tuning curves, macular and lens pigmentation, and on and on. While there may be principled grounds for saying that some such variants represent the stimulus color erroneously, there remains significant interpersonal variation among perceivers who pass standard tests for normal color vision; as such, it would be objectionably ad hoc to treat these variants as systematically misrepresenting the colors of objects. The relationalist can hold that all of these variants are veridical, thereby avoiding undue skepticism and $a d$ hoc stipulation, by holding that the stimulus exemplifies (simultaneously, all over) one color with respect to perceiver $S_{1}$, another color with respect to perceiver $S_{2}$, and so on.

Intrapersonal: The chromatic effects of a single stimulus on a single perceiver vary widely as a function of many parameters of the viewing circumstance - surround, lighting, viewing angle, viewing distance, state of adaptation of the perceiver, and on and on. While there may be principled grounds for saying that some such variants represent the stimulus color erroneously, there remains significant perceptual variation within ecologically normal circumstances; as such, it would be objectionably ad hoc to treat these variants as systematically misrepresenting the colors of objects. The relationalist holds that all of these variants are veridical, thereby avoiding both undue skepticism and ad hoc stipulation, by holding that the stimulus exemplifies (simultaneously, all over) one color with respect to circumstance $C_{1}$, another color with respect to circumstance $C_{2}$, and so on. 
It is a virtue of color relationalism that it permits a uniform diagnosis and treatment of the apparently structurally similar types of variation, and brings them all under a common theoretical umbrella.

Combining these considerations, and ignoring many niceties (also assuming that each perceiver falls in exactly one relevant perceiver type), the relationalist proposal is that colors are constituted in terms of relations to both perceivers and perceptual circumstances. Thus, on this view, colors are not monadic properties like red or green, but rather relational properties like red for $S_{1}$ in $C_{1}$ or green for $S_{2}$ in $C_{2}{ }_{3}^{3}$

\section{Part II: A Relationalist's Guide to Representation, Ontology, and Phenomenology}

Part II of The Red and the Real is devoted to showing that, contrary to what many have thought, the relationalist account of color properties argued for in Part I allows for satisfactory treatments of a range of issues about the linguistic and mental representation of color, ontology, and phenomenology.

The initial worry about linguistic and mental representation is that the surface form of ordinary color attributions ('that tomato is red', 'that beer is yellow') don't contain overt specifications of perceiver and circumstance parameters. Consequently, it appears that ordinary color attributions fail to make contact with the inventory of properties provided by relationalism.

This problem can be solved by two independently plausible ideas. The first is that ordinary color predicates pick out "coarse-grained colors" - relational properties analogous to the fine-grained colors discussed in Part I, but whose parametric positions are filled by relatively coarse-grained specifications of perceivers and perceptual conditions. This idea allows that the contents expressed by ordinary uses of color predicates need not attain the high levels of determinacy of the fine-grained colors; it simultaneously allows that the contents expressed by ordinary uses of color predicates can be multiply instantiated. The second independently plausible idea needed is a version of semantic contextualism for color language - roughly, that contextual enrichment adds to the information overtly encoded in ordinary color attributions. Combining these two ideas results in the view that the predicate 'is yellow', as uttered in context $K$, expresses the property yellow for the perceivers relevant in context $K$ under the perceptual circumstances relevant in context $K$. (Similarly for other color predicates.) Besides allowing for a solution to the initial puzzle described above, this view also paves the way for relationalist responses to an array of otherwise troublesome objections against relationalism having their source in our ordinary thought and talk about color - e.g., the worry that relationalism legitimates more color attributions than we

\footnotetext{
${ }^{3}$ There are, of course, a number of ways of resisting this argumentative pattern. One of the additional major tasks of Part I, which must go undiscussed here for reasons of space, is a critical review of these possible lines of resistance.
} 
would ordinarily accept, that it is overly permissive in the color attributions it licenses, and that it precludes errors of color representation.

I turn next to three ontological objections against relationalism: that the view proliferates colors too widely, that it amounts to a form of color irrealism, and that it is so revisionary as to count as an error theory. It seems to me that the proliferation concern is exaggerated. While there may be reason to fear a proliferation of intrinsic, fundamental properties, the sort of proliferation of relational properties to which color relationalism is committed is ontologically unproblematic. You have a height in inches less than uncountably many real numbers, and so bear uncountably many relations to the individual numbers; but because these relations add nothing to the fundamental ontology of the world, their proliferation is ontologically innocent. I claim that the proliferation of colors constituted by relations to different perceivers and circumstances is similarly innocuous. Turning to irrealism, I accept that some technical understandings of the term might extend to relationalism. Nonetheless, I insist that relationalism does not entail that colors fail to exist or are unexemplified (a view that I take to be, at best, a position of last resort), and so avoids the objectionably revisionary sense of the label. Finally, I consider the accusation that relationalism is an error theory of color in the (Mackie-inspired) sense that it contravenes anti-relational presuppositions inherent in our naïve color concepts. This worry, too, strikes me as unpersuasive. For one thing, there is empirical evidence that ordinary thinkers lack the robust anti-relational presuppositions the worry claims they have. For another, even if thinkers do hold anti-relational beliefs, there is little reason to accept that such beliefs are analytically connected to color concepts; but if not, then their contravention by relationalism does not make that view an error theory.

The final cluster of issues discussed in Part II concerns phenomenology. Specifically, it addresses the oft-made objection that ordinary color phenomenology presents colors as being non-relational, hence that (barring an error theory of color experience) we should side with the manifest data of color phenomenology and so reject color relationalism.

Before I can answer this objection directly, I need to say how (and whether) color phenomenology can bear on the relationality or non-relationality of colors, as the objection presupposes. I claim that, on a narrow construal, phenomenology is unlikely to have much to say about the relationality of the properties it represents, or on any other matters of their metaphysical constitution. On the other hand, if we construe phenomenology more broadly - as allowing for comparison between phenomenal representations, together with ratiocinative reflection, then I believe it can speak to the issue at hand. To see this point, notice how the determination that motion properties are constituted in terms of relations to reference frames crucially involves comparison: an essential part of the (defeasible) case for relationality involves realizing that the apparent velocity of $x$ relative to frame $F_{1}$ is different from the apparent velocity of $x$ relative to frame $F_{2}$, and that neither the representation relative to $F_{1}$ nor the representation relative to $F_{2}$ is veridical at the expense of the other. Similarly, we can bring phenomenological evidence to bear (defeasibly) on the 
relationality of color properties, but only by applying analogously comparative methods to color phenomenology. Thus, we can notice that the phenomenal representation of $x^{\prime}$ s color relative to $S_{1}$ in $C_{1}$ is different from the phenomenal representation of $x$ 's color relative to $S_{2}$ in $C_{2}$; and if (as I argued in Part I) we have reason to deny that either phenomenal representation is veridical to the exclusion of the other, then that phenomenal evidence can form part of a defeasible, empirical argument for the relationality of colors. That, I claim, is exactly the situation we find. I conclude, therefore, that phenomenology can bear on the relationality of color when treated correctly - and that, so treated, phenomenology tells in favor of color relationalism rather than against it.

\section{Part III: Role Functionalism}

Having argued that color relationalism is both true and conducive to attractive positions about allied issues, I turn in Part III to laying out and defending the more specific form of color relationalism that I favor: role functionalism.

Role functionalism about color says that colors are identical with functional roles connecting lights, surfaces, and the like, to visual systems. Specifically, role functionalism claims that red for $S$ in $C$ is the functional role of disposing its bearers to look red to $S$ in $C$, that green for $S$ in $C$ is the functional role of disposing its bearers to look green to $S$ in $C$, and so on for the other colors.

But how should we understand those functional roles? I claim that $x$ looks red/green/etc. to $S$ in $C$ iff, by visually attending to $x$ in $C, S$ is appropriately caused in $C$ to have an experience of red/green/etc. And what is an experience of red/green/etc.? By this I mean a type of mental states of subjects - namely, that type of mental state whose tokens are the typical effects of those subjects' attending to things that are red/green/etc. (say, ripe raspberries/limes/etc.). But I claim that role functionalists, qua role functionalists, need not say much more about the metaphysical constitution of the relevant mental types. For it seems to me that color role functionalism can be coherently combined with a wide variety of theories of those mental types, including functionalist, representationalist, type identity, and primitivist theories. I see the detachability of color functionalism from views about the metaphysics of color experience as a benefit of the former, since it means that proponents of many different answers to the latter question can accept the position.

Setting out role functionalism about color in this way invites comparison to an alternative view: realizer functionalism. Both views about color are modeled on analogous and familiar positions about mental states. Role functionalists about the mental include Putnam (1967); Block and Fodor (1972); realizer functionalists include Armstrong (1968, 1970); Lewis (1972). Role and realizer functionalists agree that their targets should be understood in terms of the functional roles they play; but, whereas role functionalists identify the targets with those functional roles themselves, realizer functionalists identify the targets with (not the roles, but) the occupants of the roles. Thus, for example, 
role functionalists about mental states might identify the property being $a$ belief with a type of functional role in the mental economy of a thinker, while realizer functionalists would identify being a belief with (not the functional role, but) whatever material micro-constitutions, primitive extra properties, etc. they take to (contingently) play the relevant functional role. Likewise, role functionalists about color identify red for $S$ in $C$ with the functional role of disposing its bearers to look red to $S$ in $C$, while realizer functionalists identify red for $S$ in $C$ (not with the functional role of disposing its bearers to look red to $S$ in $C$, but) with whatever material micro-constitutions, reflectance properties, primitive extra properties, etc. they take to (contingently) play that functional role.

While this difference has many interesting consequences, one of the most important given the discussion so far is that realizer functionalism is not a species of color relationalism. For although realizer functionalists accept that the functional roles relevant to the metaphysics of colors are those of disposing things to look red/green/etc. to $S$ in $C$, and though those roles crucially implicate relations to perceivers, realizer functionalists take these roles to be inessential to the colors: they hold that the colors are the realizers that (contingently, de re) happen to play the roles. And since there is no reason to assume that the realizers of such relationally constituted roles must be relational, realizer functionalists need not be color relationalists.

There are (at least) three serious costs of realizer functionalism. The first is that realizer functionalism fails to account for what is necessarily common to distinct instances of a given color. Of course, both role and realizer functionalisms allow one to say that distinct instances of a color share a functional role. But since realizer functionalists take having that role to be (de $r e$ ) inessential to each color instance, they can only explain what is common to the instances of a given color in terms of something they take to be inessential to that color, and only contingently exemplified by its instances. I claim an adequate metaphysics of colors should allow more than this, lest it devolve into a mere list. My second concern about realizer functionalism is that it is unable to secure modally necessary similarity and exclusion relationships between colors, such as that orange is necessarily more similar to red than to green, or that necessarily no shade of yellow is a shade of blue. This is because the best explanations of such relationships turn on facts about how perceivers' visual systems represent colors. Since the material structures realizer functionalists identify with colors are only contingently connected to perceivers (/their visual systems), realizer functionalists are committed to holding that colors have their similarity and exclusion relations only contingently. My third objection to realizer functionalism is that the view entails that color terms refer non-rigidly to whatever happens to realize the relevant functional roles; but on standard tests, color terms appear to refer rigidly. I believe these three problems are serious concerns for realizer functionalism, and that role functionalism escapes each of them.

However, there remains a major obstacle to role functionalism that must be overcome before the latter view can be taken seriously: many have argued 
that functional roles cannot do the causal work that colors uncontroversially do (viz., the work of causing their bearers to look colored to perceivers). Perhaps the most important motivation for this claim is the idea that, since every particular exemplification of the relevant functional role occurs coincidently with an exemplification of a realizer of that role, and since it is common ground that the realizer is causally sufficient for the eventual effects in perceivers, according causal efficacy to the role property threatens causal overdetermination.

Ultimately, however, I find this argument unpersuasive. For it seems to me that the kind of causal efficacy our intuitions warrant us in according to the colors is a sort that is not competitive with - indeed, one that can be constituted by - the causal efficacy of its realizers (here I adapt a proposal of Yablo (1992)). To see the idea, consider the following example. Suppose a bell will ring if the mass on a platform exceeds $1000 \mathrm{~kg}$, and that an object of $1037 \mathrm{~kg}$ is placed on that platform. I take it that the bell's ringing can be causally explained either by citing the object's property of being over $1000 \mathrm{~kg}$ or its modally more determinate property of being $1037 \mathrm{~kg}$. Likewise, I claim, we can causally explain the effects of a colored patch on a visual system either by citing its role property or the its modally more determinate realizer of that role. Of course, on particular occasions we may have preferences between citing the modally more determinate or modally less determinate properties in giving a causal explanation. But I take these preferences to reflect our occasion-bound pragmatic explanatory purposes; I don't see why they should be taken to show (as the objection presupposes) that one putative explanans is metaphysically prior to another, or that one causally/explanatorily precludes the other from serving in an equally acceptable causal explanation.

As I see matters, then, the realizer functionalist view faces serious unanswered threats, while the threats against role functionalism can be answered. Therefore, it seems to me that we would be wise to favor the latter view over the former.

In the final chapter of Part III I attempt to extend the motivation for role functionalism by comparing it against several ostensibly competing forms of color relationalism: classical color dispositionalism, the "enactive" ecological relationalism of Thompson (1995), and the sensory classificationist view of Matthen (2005). I argue that each of these alternative views is untenable. First, I contend that, depending on how one understands the metaphysics of dispositions, dispositionalism is either vulnerable to fatal objections concerning the relation between dispositions and their categorical bases or else coincides with the role functionalist view I advocate. Second, I claim that the enactive ecological view cannot accommodate the observed multiple realization of colors, and that it has either implausibly idealist implications, or else mislocates the dispositions to look colored on properties, rather than perceived objects. Finally, I argue that the sensory classificationist view depends on implausibly tight connections between organisms' sensory 
classifications of distal stimuli and the epistemic and non-epistemic actions they afford.

While these considerations do not (and cannot) exhaust the range of possible relationalist alternatives, in my view they motivate rejecting the relationalist alternatives to role functionalism that have received the most philosophical support and interest. Accordingly, until a more attractive form of relationalism emerges, these considerations suggest that relationalists about color should be role functionalists about color.

\section{Conclusion}

The separate Parts of The Red and the Real comprise a larger argument for role functionalism about color. Parts I and II are intended to show that we should accept the most plausible relationalist account available, and that the theoretical costs of doing so are modest; and Part III is intended to show that role functionalism is, as it turns out, the most plausible relationalist account available.

I believe color relationalism and role functionalism have much to recommend them. For all I know, they might even be true.

\section{References}

Armstrong, D. (1968). A Materialist Theory of the Mind. Routledge, London.

Armstrong, D. M. (1970). The nature of mind. In C. V. Borst, editor, The Mind/Brain Identity Theory. MacMillan, London. Reprinted in Block (1980), 191-199.

Block, N., editor (1980). Readings in the Philosophy of Psychology, Volume 1. Harvard University Press, Cambridge, Massachusetts.

Block, N. and Fodor, J. A. (1972). What psychological states are not. The Philosophical Review, 81(2), 159-182. Reprinted in Block (1980), 237-250.

Lewis, D. (1972). Psychophysical and theoretical identitifications. Australasian Journal of Philosophy, 50(3), 249-258. Reprinted in Block (1980), 207-215.

Matthen, M. (2005). Seeing, Doing, and Knowing: A Philosophical Theory of Sense Perception. Oxford University Press, Oxford.

Putnam, H. (1967). The nature of mental states. In W. H. Capitan and D. D. Merrill, editors, Art, Mind, and Religion, pages 37-48. University of Pittsburgh Press, Pittsburgh. Reprinted in Block (1980), 223-231.

Thompson, E. (1995). Colour Vision: A Study in Cognitive Science and the Philosophy of Perception. Routledge, New York.

Yablo, S. (1992). Mental causation. Philosophical Review, 101, 245-280. 